\title{
Corrosion par mélange des eaux
}

Par Alfred Bögli, Hitzkirch (Suisse)

Avec planches 15(1)-17(3)

Le karst se distingue des autres types de paysage par la prépondérence du drainage souterrain. Dans le stade de maturité, le karst peut absorber à peu près toutes les précipitations. Par contre la nappe d'eau karstique monte parfois jusqu'à la surface, par exemple dans les poljés. Au Hölloch (Suisse centrale) la différence atteint jusqu'à $150 \mathrm{~m}$. La nappe d'eau est une surface piézomètrique. Le drainage souterrain est la conséquence de la solubilité de la roche, notamment des carbonates. La connaisssance approfondie des procédés de dissolution est nécessaire pour comprendre la formation et l'évolution des galeries souterraines.

\section{$1^{\circ}$ - La conception actuelle de la dissolution des carbonates et leur conséquence}

Normalement les réseaux souterrains prennent naissance par des joints capillaires ou à peu près capillaires, soit sur les plans de stratification (p.e. la plupart des $78 \mathrm{~km}$ de galeries du Hölloch), soit par des diaclases. Ils ont été d'abord élargis par corrosion. La dissolution des carbonates n'est d'ailleurs pas un phénomène physique mais chimique et suit la réaction bien connue

$$
\mathrm{CO}_{3} \mathrm{Ca}+\mathrm{CO}_{2}+\mathrm{H}_{2} \mathrm{O} \leftrightharpoons \mathrm{Ca} \cdot+2\left(\mathrm{CO}_{3} \mathrm{H}\right)^{\prime} .
$$

La dissolution exige la présence de $\mathrm{CO}_{2}$ et il est necessaire que la nature le fournisse. A la condition que l'équilibre dans l'eau soit atteint, il y a trois possibilités:

a - La concentration du $\mathrm{CO}_{2}$ dans l'eau correspond à la teneur en $\mathrm{CO}_{2}$ dans l'air. La concentration reste constante et rien ne change.

b- La concentration ne correspond pas à celle dans l'air. Si elle est trop grande, il se dégage du $\mathrm{CO}_{2}$ et il en résulte des dépôts de $\mathrm{CO}_{3} \mathrm{Ca}$ sous forme de concrétions. S'il y a un défaut de $\mathrm{CO}_{2}$ dans l'eau, elle en adsorbe et dissout du carbonate jusqu'à ce que l'équilibre avec l'air soit réalisé. 
c - L'eau de la zone phréatique et des conduites forcées n'a pas de contact avec l'air, ni celui de l'atmosphère ni celui du sol, et forme un système chimique clos. Rien ne change, et par conséquent il n'y a pas de dissolution du calcaire. Les joints et fissures primaires sont normalement capillaires ou au moins très étroits et ils sont ou secs ou remplis d'eau. Sauf sur une partie, au commencement de la fissure, leur contenu est sans surface libre et forme un système chimique clos, et par suite sans possibilité de corrosion. Cela est très important, car l'élargissement des joints primaires ne se fait que par dissolution du calcaire.

La source la plus importante du $\mathrm{CO}_{2}$ est celui de l'atmosphère et celui du sol. Pour atteindre l'équilibre, il faut quelques heures avec des températures au-dessus de $20^{\circ} \mathrm{C}$, plusieurs jours au-dessous de $5^{\circ} \mathrm{C}$, pourvu qu'il y ait une surface libre, soit dans un lac souterrain, soit dans la circulation torrentielle. La zone phréatique, les conduites forcées et les siphons longs sont exclus de cette corrosion.

Dans l'eau sans surface libre on trouve assez fréquemment des matières organiques. Par oxydation elles se transforment en $\mathrm{CO}_{2}$ biogène. La présence d'oxygène est indispensable. Les microbes jouent un rôle important. Pour le transport des matières organiques, il faut déjà un réseau assez élargi. Il n'est pas question d'un élargissement des joints primaires par le $\mathrm{CO}_{2}$ biogène qui s'est formé dans l'eau même. Mais une possibilité de corrosion restreinte par cette voie est donné pour les conduites forcées et la partie supérieure de la zone phréatique. Notons que la vitesse de l'oxydation est très lente aux températures au-dessous de $10^{\circ} \mathrm{C}$. Au Hölloch, nous avons trouvé des argiles pleistocènes (würmiennes ou antéwürmiennes) avec des teneurs en matières humiques allant jusqu'à 1,3\%. La moyenne actuelle de la température est de $5 \frac{1}{2}{ }^{\circ} \mathrm{C}$.

L'eau agressive peut emporter le $\mathrm{CO}_{2}$ du dehors et provoque une corrosion peu considérable. Dans les rivières souterraines, le $\mathrm{CO}_{2}$ libre pénètre assez loin dans l'intérieur de la terre, tandis que dans les joints primaires et dans les diaclases étroites l'effet ne dépasse guère quelques centimètres.

Ce résumé montre que l'élargissement des joints capillaires et des diaclases étroites ne s'explique nullement par la conception actuelle de la dissolution des carbonates. Aussi l'élargissement par corrosion des conduites forcées n'est possible que sous des conditions très restreintes, ce qui vaut aussi pour la partie supérieure de la zone phréatique. La dimension est d'ailleurs peu considérable. La zone phréatique inférieure est exclue de cette corrosion. Mais c'est un fait que dans la zone phréatique il y a assez souvent des cavités, qui ne 
s'expliquent pas par un abaissement tectonique. Autrement dit: La naissance de la plupart des grottes ne s'explique pas par la conception actuelle de la dissolution des carbonates.

\section{$2^{\circ}$ - La corrosion par mélange des eaux}

Entre le bicarbonate de calcium et le $\mathrm{CO}_{2}$ équilibrant il y a des des relations. Tillmans a établi un relevé des valeurs du $\mathrm{CO}_{2}$ eq. A l'EAWAG (Eidgenössische Anstalt für Wasserversorgung, Abwasserreinigung und Gewässerschutz) à Zurich, on a trouvé que les valeurs du $\mathrm{CO}_{2}$ eq. de Tillmans pour des concentrations de $\mathrm{CO}_{3} \mathrm{Ca}$ au-delà de $200 \mathrm{mg}$ au litre étaient trop grandes. Zehender, Stumm et Fischer ont dressé une nouvelle formule et ils ont fixé les valeurs selon le tableau I.

Tableau 1

$\mathrm{Co}_{2}$ eq. d'après Tillmans (modifié par Bögli) et d'après Zehender, Stumm et Fischer à $17^{\circ} \mathrm{C}$, en $\mathrm{mg} / \mathrm{l}$

\begin{tabular}{|c|c|c|c|c|c|}
\hline $\begin{array}{l}\mathrm{mg} / \mathrm{l} \\
\mathrm{O}_{3} \mathrm{Ca}\end{array}$ & Tillmans & $\begin{array}{c}\text { Zehender, } \\
\text { Stumm et } \\
\text { Fischer } \\
\mathrm{CO}_{2} \text { eq. }\end{array}$ & $\begin{array}{c}\mathrm{mg} / \mathrm{l} \\
\mathrm{CO}_{3} \mathrm{Ca}\end{array}$ & Tillmans & $\begin{array}{c}\text { Zehender, } \\
\text { Stumm et } \\
\text { Fischer } \\
\mathrm{CO}_{2} \text { eq. }\end{array}$ \\
\hline 10 & 0 & 0 & 190 & 13,38 & 13,14 \\
\hline 40 & 0,41 & 0,16 & 200 & 15,97 & 15,19 \\
\hline 50 & 0,58 & 0,30 & 210 & 18,92 & 17,44 \\
\hline 60 & 0,80 & 0,50 & 220 & 22,29 & 19,98 \\
\hline 70 & 1,08 & 0,80 & 230 & 26,07 & 22,55 \\
\hline 80 & 1,41 & 1,14 & 240 & 30,27 & 25,41 \\
\hline 90 & 1,81 & 1,60 & 250 & 34,91 & 28,52 \\
\hline 100 & 2,27 & 2,14 & 260 & 39,99 & 31,87 \\
\hline 110 & 2,82 & 2,80 & 270 & 45,47 & 35,43 \\
\hline 120 & 3,46 & 3,59 & 280 & 51,30 & 39,27 \\
\hline 130 & 4,23 & 4,50 & 290 & 57,49 & 43,30 \\
\hline 140 & 5,15 & 5,54 & 300 & 64,0 & 47,7 \\
\hline 150 & 6,26 & 6,73 & 320 & 77,9 & 57,2 \\
\hline 160 & 7,61 & 8,08 & 340 & 93,0 & 67,8 \\
\hline 170 & 9,22 & 9,60 & 360 & 109,4 & 79,6 \\
\hline \multirow[t]{2}{*}{180} & 11,12 & 11,28 & 380 & 126,9 & 92,5 \\
\hline & & & 400 & 145,9 & 106,8 \\
\hline
\end{tabular}

La courbe d'équilibre pour l'hydrocarbonate de calcium sépare la zone de précipitation de celle de l'eau agressive vis-à-vis du calcaire (graphique 1). Une ligne droite qui relie deux points sur cette courbe 
traverse toujours la zone de l'eau agressive. Cette droite est la somme de tous les mélanges possibles entre les deux eaux $W_{1}$ et $W_{2}$. Il est évident que chaque fois que l'on mêle des eaux d'une teneur différente en $\mathrm{CO}_{3} \mathrm{Ca}$ il en résulte du $\mathrm{CO}_{2}$ libre. Le mélange supposé est de 1 pour 1 . On peut trouver par calcul la somme de $\mathrm{CO}_{3} \mathrm{Ca}$ dissout en supplément, ce qui est un peu compliqué à cause du $\mathrm{CO}_{2}$ eq.

Tableau 2

$\mathrm{CO}_{3} \mathrm{Ca}$ dissout en supplément d'après les valeurs de Tillmans $\left(17^{\circ} \mathrm{C}\right)$

\begin{tabular}{|c|c|c|c|c|c|c|}
\hline \multicolumn{2}{|c|}{$\begin{array}{l}\text { Concentration des } \\
\text { composantes en } \\
\mathrm{CO}_{3} \mathrm{Ca}\end{array}$} & \multicolumn{5}{|c|}{$\begin{array}{l}\text { Proportions du mélange et quantité de } \\
\mathrm{CO}_{3} \mathrm{Ca} \text { dissout en supplément en mg/l. }\end{array}$} \\
\hline $\mathrm{W}_{1} \mathrm{mg} / \mathrm{l}$ & $\mathrm{W}_{2} \mathrm{mg} / \mathrm{l}$ & $9 / 1$ & $3 / 1$ & $1 / 1$ & $1 / 3$ & $1 / 9$ \\
\hline 11,5 & 125,0 & 0,6 & 1,1 & 1,8 & 1,2 & 0,75 \\
\hline 11,5 & 221,6 & 4,5 & 10,1 & 15,9 & 12,9 & 6,0 \\
\hline 11,5 & 329,6 & 17,2 & 38,8 & 50,1 & 29,1 & 12,6 \\
\hline 73,9 & 125,0 & 0,2 & 0,3 & 0,5 & 0,4 & 0,2 \\
\hline 73,9 & 221,6 & 3,3 & 7,5 & 10,5 & 7,7 & 3,7 \\
\hline 73,9 & 329,6 & 14,9 & 30,2 & 33,7 & 20,4 & 8,9 \\
\hline 125,0 & 170,5 & 0,4 & 0,6 & 1,0 & 0,6 & 0,3 \\
\hline 125,0 & 272,7 & 5,5 & 11,0 & 13,3 & 8,8 & 3,9 \\
\hline 125,0 & 358,0 & 14,0 & 25,7 & 26,3 & 16,1 & 7,0 \\
\hline 170,5 & 272,7 & 2,7 & 5,4 & 6,6 & 4,3 & 1,9 \\
\hline 170,5 & 301,4 & 4,3 & 8,5 & 9,6 & 6,1 & 2,7 \\
\hline 170,5 & 358,0 & 8,6 & 15,5 & 16,1 & 10,2 & 4,5 \\
\hline 272,7 & 358,0 & 1,0 & 2,1 & 2,6 & 1,9 & 0,8 \\
\hline
\end{tabular}

Il est plus simple de chercher le résultat par graphique. Nous prenons $\mathrm{T}$ comme remplaçant pour le mélange. On y trouve directement la teneur en $\mathrm{CO}_{3} \mathrm{Ca}$ et en $\mathrm{CO}_{2}$. La partie gauche du $\mathrm{CO}_{2}$ jusqu'à la courbe est le $\mathrm{CO}_{2}$ eq. du mélange, le reste du $\mathrm{CO}_{2}$ libre, représenté par la distance CT. La partie BT est employée pour la dissolution du $\mathrm{CO}_{3} \mathrm{Ca}$ supplémentaire. $50 \mathrm{mg}$ de $\mathrm{CO}_{3} \mathrm{Ca}$ consomment $22 \mathrm{mg}$ de $\mathrm{CO}_{2}$. Cela nous permet de fixer l'inclinaison de la ligne TA. Cette direction reste la même pour tous les mélanges possibles. $\mathrm{CB}$ répond au $\mathrm{CO}_{2}$ eq. additionnel.

L'importance de la corrosion par mélange des eaux pour l'interprétation des cavités est très grande. Elle explique la corrosion des galeries de toute dimension à toutes les profondeurs. La corrosion par mélange des eaux n'agit pas seulement dans l'écoulement libre, mais aussi et surtout dans les conduites forcées et dans la zone phréatique, 
donc dans les domaines qui ne sont pas ou peu attaqués par la corrosion dite normale. C'est un point caractéristique de l'hydrographie karstique que les conduits sont très souvent indépendants l'un de l'autre. A cause de cela, la dureté de l'eau varie d'un conduit à l'autre. Cette différence est la base de toute la corrosion par mélange des eaux. En voici un exemple:

L'eau d'une moraine s'infiltre dans deux diaclases du calcaire. Elle a une teneur supposée en carbonate de chaux de 272,7 $\mathrm{mg}$ au litre. Dans la première fissure elle poursuit son chemin en conduite forcée et ne change pas de concentration. Dans la deuxième elle atteint une galerie et coule en surface libre. $\mathrm{Le}_{\mathrm{CO}_{2}}$ se dégage en partie et provoque la précipitation de $\mathrm{CO}_{3} \mathrm{Ca}$ sous forme de concrétions. Après un certain temps, l'eau contient encore $125 \mathrm{mg}$ de carbonate par litre et entre enfin dans une fissure. Dans la zone phréatique elle rejoint la première partie de l'eau qui n'a pas changé de concentration. La différence est importante et provoque par un mélange des 1 pour 1 une dissolution supplémentaire de 13,3 mg de calcaire par litre. C'est ainsi que de grandes cavités sans accès remarquable peuvent prendre naissance.

Dans le karst vert l'eau contient ordinairement beaucoup de carbonate de chaux. Si l'on mélange ces eaux d'une haute teneur en bicarbonate, elles sont capables de dissoudre le calcaire (tableau II). Ce fait est d'une grande importance pour le développement du karst. Cela explique la situation paradoxale que dans le karst vert il y a souvent de grandes grottes. S'il s'y ajoute des parties de karst nu comme c'est fréquement le cas dans les Alpes ou dans les pays méditerrannéens, la différence des concentrations peut devenir très grande. C'est là où se trouvent les plus grandes cavités, par exemple le Hölloch $(78 \mathrm{~km})$ et la Eisriesenwelt $(42 \mathrm{~km})$ en Europe. N'oublions pas que l'érosion mécanique joue un rôle important dès le moment où l'élargissement a atteint une certaine dimension.

\section{$3^{\circ}$ - La corrosion par refroidissement}

A l'aide de la formule de Zehender, Stumm et Fischer il est possible de mesurer l'effet de la corrosion de refroidissement et celui de la corrosion par mélange des eaux de température différente.

L'eau étant en l'équilibre de $\mathrm{CO}_{2}$ à une certaine température ne l'est plus si celle-ci varie. Quand elle monte, le $\mathrm{CO}_{2}$ eq. ne suffit plus pour tenir le $\mathrm{CO}_{3} \mathrm{Ca}$ en solution: précipitation. Quand elle s'abaisse, le $\mathrm{CO}_{2}$ eq. diminue et il en résulte un surplus de $\mathrm{CO}_{2}$ libre, ce qui provoque de la corrosion (graphique 2). 
Tableau 3

Le $\mathrm{CO}_{2}$ eq. en fonction de la température

\begin{tabular}{c|rrrrrrr}
\hline $\mathrm{CO}_{3} \mathrm{Ca}$ & \multicolumn{7}{|c}{$\mathrm{CO}_{2}$ eq. en mg par litre } \\
$\mathrm{mg} / \mathbf{l}$ & $0^{\circ} \mathrm{C}$ & \multicolumn{1}{c}{$3^{\circ} \mathrm{C}$} & \multicolumn{1}{c}{$6^{\circ} \mathrm{C}$} & $10^{\circ} \mathrm{C}$ & $15^{\circ} \mathrm{C}$ & $20^{\circ} \mathrm{C}$ & $24^{\circ} \mathrm{C}$ \\
\hline $\mathbf{1 2 0}$ & 1,94 & 2,15 & 2,40 & 2,78 & 3,33 & 4,00 & 4,62 \\
$\mathbf{1 6 0}$ & $\mathbf{4}, 38$ & $\mathbf{4}, 86$ & 5,40 & 6,26 & 7,50 & 9,00 & 10,41 \\
200 & 8,23 & 9,13 & 10,15 & $\mathbf{1 1 , 7 6}$ & 14,11 & 16,93 & 19,57 \\
240 & 13,77 & 15,28 & 16,99 & 19,69 & 23,61 & 28,31 & 32,74 \\
280 & 21,29 & 23,60 & 26,23 & 30,40 & 36,46 & 43,75 & 50,56
\end{tabular}

Tableau 4

Carbonate de chaux supplémentaire par refroidissement de $\left(\mathrm{T}_{2}-\mathrm{T}_{1}\right)^{\circ} \mathrm{C}$ (valeurs rapprochées)

\begin{tabular}{|c|c|c|c|c|c|c|}
\hline \multirow{2}{*}{$\begin{array}{c}\mathrm{CO}_{3} \mathrm{Ca} \\
\mathrm{mg} / \mathrm{l}\end{array}$} & \multicolumn{6}{|c|}{$\mathrm{CO}_{3} \mathrm{Ca}$ supplémentaire } \\
\hline & $6-0^{\circ}$ & $10-6^{\circ}$ & $15-10^{\circ}$ & $15-6^{\circ}$ & $20-15^{\circ}$ & $24-20^{\circ}$ \\
\hline 120 & 1,0 & 0,9 & 1,2 & 2,1 & 1,5 & 1,4 \\
\hline 160 & 2,3 & 1,9 & 2,7 & 4,6 & 3,2 & 3,0 \\
\hline 200 & 4,2 & 3,5 & 5,0 & 8,5 & 5,9 & 5,5 \\
\hline 240 & 6,9 & 5,7 & 8,1 & 13,8 & 9,6 & 8,8 \\
\hline 280 & 10,3 & 8,5 & 12,0 & 20,7 & 14,3 & 12,9 \\
\hline
\end{tabular}

La corrosion par refroidissement se borne à une zone assez étroite à proximité de la surface. En outre, on ne la trouve que dans des climats ayant de grandes différences de température journalières ou saisonnières. A une profondeur de 10 à $25 \mathrm{~m}$, la température du sol atteint la moyenne du climat. Plus bas, elle monte d'après le gradient géothermique. Il est évident que ce type de corrosion ne se développe que dans la saison chaude.

Dans le stade de maturité du karst les proportions changent fondamentalement. Par la circulation de l'air et des eaux, la roche se rafraîchit et atteint une température qui est un peu plus haute que la moyenne de la surface. En été, on trouve dans le Hölloch partout une température de $5^{\circ} \mathrm{C}$, indépendant de l'épaisseur de la roche qui varie entre 300 et $900 \mathrm{~m}$. C'est la saison des eaux de fonte de neige et des grandes crues qui font baisser la température. En hiver la température augmente d'un seul degré par suite de la chaleur de l'intérieur de la terre. Mais cela ne suffit pas pour établir un gradient géothermique à peu près normal.

Le mélange de deux eaux de même concentration, mais de temperature différente, produit un effet de corrosion qui s'explique facile- 
ment à l'aide du tableau III. La quantité de $\mathrm{CO}_{3} \mathrm{Ca}$ dissout en supplément est petite et ne dépasse pas quelques mg au litre.

\section{$4^{\circ}$ - Les formes de la corrosion par mélange des eaux}

Les formes de référence pour la corrosion par mélange des eaux sont encore peu étudiées par suite du peu de temps passé depuis la découverte. Actuellement il est possible d'en distinguer trois types. Citons d'abord les grandes cavités de formes caractéristiques qui ont pris naissance au-dessous de la nappe d'eau karstique et dans les conduites forcées de la zone vadose. Le plus grand diamètre se trouve normalement en amont, là où deux conduits d'eau s'entrecroisent sur un plan qui ne dépasse pas trop les $100 \%$ de pente. Ces deux eaux se mêlent et provoquent une corrosion. En aval la concentration du $\mathrm{CO}_{2}$ libre diminue par suite de la corrosion du calcaire et en même temps le diamètre de la galerie qui succède. Très souvent ces cavités prennent la forme de salles, dont l'accès par les affluents est assez pénible, sinon impossible. A première vue, il semble impossible d'expliquer comment, à partir de ces petits affluents, un espace vide d'une telle étendue à pu se former.

Si la partie noyée d'une diaclase plus ou moins perpendiculaire croise un plan de stratification noyé lui aussi, la corrosion n'agit pas seulement sur ce plan mais aussi dans la verticale. Le résultat est une forme de cloche ou même un dôme, très souvent modifié par décollement à la voûte. Il est très probable que la plupart des salles où plusieurs galeries s'entrecroisent, ainsi que des puits en forme de cloche doivent leur forme avant tout à la corrosion par mélange des eaux.

La galerie en forme de cul-de-šc est le répresentant du deuxième type. Très souvent, on trouve des galeries elliptiques sur les joints de stratification. Elles finissent en amont par une abside taillée dans la roche où entrent des petits canaux d'accès ne dépassant parfois pas même quelques centimètres de diamètre. La galerie ne finit donc pas par un amincissement jusqu'au dimension d'une fissure. L'eau qui y arrive se mêle dans l'abside et commence à corroder. En continuant son cours le $\mathrm{CO}_{2}$ libre diminue de plus en plus. C'est en somme la même situation que dans les grandes salles du premier type, sauf que la quantité d'eau y est beaucoup plus petite.

Si un ruisseau souterrain arrive à une galerie qui mène beaucoup d'eau, il se forme une courte galerie en cul-de-sac. La longueur ne dépasse guère $10 \mathrm{~m}$. Mais elle est assez large à la base. Elle ressemble parfois à une niche. 
Notons ici les labyrinthes qui sont parfois des modifications des galeries en cul-de-sac. Sur les plans de stratification l'eau coule souvent en filons avec des liaisons transversales. Au croisement avec une fissure pleine d'eau, chaque filon provoque la formation d'une petite galerie en cul-de-sac, tandis qu'en aval l'eau forme un canal d'écoulement elliptique à l'aide du $\mathrm{CO}_{2}$ libéré par le mélange. Le côté où l'eau arrive montre pendant la première phase une forme en guirlande, forme d'ailleurs assez fréquente dans les grottes. Il est possible que ces parois en guirlande, parfois avec des piliers, soient aussi des formes de référence mais cela est encore à prouver. Ces niches s'accroissent le long des filons et de leurs anastomoses, et il en résulte un labyrinthe. Mais pour le moment il n'est pas question de les ranger sous les formes de référence parce qu'il y a d'autres possibilités pour leur formation.

Les marmites de corrosion sont le troisième type (photos, 1, 2, 3). Elles se trouvent surtout à la voûte et sur les parois. Quand une fissure pleine d'eau traverse une galerie inondée, la corrosion par mélange des eaux est inévitable. Normalement l'eau de la diaclase contient plus de bicarbonate parce que les crues qui envahissent les galeries apportent de l'eau peu concentrée. Malgré tout, les cas inverses sont assez fréquents dans les grottes se trouvant à la proximité de la surface. Les marmites de corrosion sont un équivalent des galeries en cul-de-sac. Mais elles ne se forment que si la différence entre les arrivées d'eau est très grande, tandis que dans les deux premiers types, les arrivées d'eau sont du même ordre de grandeur.

Les marmites de corrosion sont très typiques dans les galeries de forme hydrique. On y trouve assez souvent aussi des concrétions, ce qui est normal. L'eau des diaclases a parfois une teneur en bicarbonate élevée. Si elle entre dans une galerie sèche, elle dégage du $\mathrm{CO}_{2}$ en précipitant des concrétions. Il semble paradoxal que la même eau puisse corroder le calcaire en entrant dans une galerie inondée, tandis qu'elle produit des concrétions dans les galeries sèches (photo 3).

Il y a sûrement d'autres formes de référence, mais dans l'état actuel des recherches, il est encore trop tôt pour les nommer.

\section{RESUMÉ}

Les cavités karstiques sont avant tout dues à la corrosion. D'après la formule sommaire bien connue, il faut toujours une arrivée de $\mathrm{CO}_{2}$. Ce type de dissolution nous explique seulement la corrosion en circulation libre et, sous réserve, en conduite forcée dans la zone vadose. Toute corrosion dans la zone phréatique en est exclue, sauf quelques cas assez rares dans la partie supérieure. 
La corrosion par mélange des eaux de teneur différente en bicarbonate intéresse toute la zone karstifiée, des parties les plus basses du domaine phréatique jusqu'à la surface. On peut y ajouter la corrosion provoquée par le refroidissement et le mélange des eaux de températures différentes. Hors quelques cas exceptionnels, (sources thermiques p. e.) l'effet en est très réduit.

\section{ZUSAMMENFASSUNG}

Die unterirdische Entwässerung ist ein Kennzeichen des Karstes. Sie ist eine Folge der subterranen Korrosion. Die konventionelle Auffassung von der Kalklösung verlangt die Zufuhr von $\mathrm{CO}_{2}$ von außen, großenteils aus der Luft, zu einem kleinen Teil aus dem Wasser durch Oxidation organischer Substanzen. Der Wirkungsraum ist die vadose Zone, vor allem sind es die Gravitationsgerinne, während die Druckgerinne nur selten auf diese Art korrodiert werden, etwa dann, wenn das zufließende Wasser noch aggressiv ist oder organische Substanzen enthält. Ähnliches gilt für die hochphreatische Zone, wenn auch noch mehr beschränkt, während in der tiefphreatischen dieser Korrosionstyp nicht mehr auftreten kann.

Mischungskorrosion tritt dann auf, wenn sich zwei verschieden konzentrierte Wasser mischen. Nach Diagramm 1 liegen alle Mischungen im Bereiche des kalkaggressiven Wassers. Das ursprüngliche $\mathrm{CO}_{2}$ eq. wird nur zu einem Teil in der Mischung gebraucht, der Rest wird frei und bewirkt Kalkkorrosion (Tab. 2). Hochkonzentrierte Wasser, wie sie im grünen Karst normal sind, können bis zu $20 \mathrm{mg}$ Kalk im Liter zusätzlich lösen. Wo sich grüner und nackter Karst durchdringen, sind die Konzentrationsdifferenzen besonders groß, und die Mischungskorrosion erreicht Werte bis zu $50 \mathrm{mg} / \mathrm{l}$ zusätzlich gelösten Kalkes, was durch die großen Höhlen solcher Regionen bewiesen wird. Mischungskorrosion wirkt von den tiefsten Bereichen der phreatischen Zone bis zur Erdoberfläche in den Druckgerinnen wie in den Gravitationsgerinnen.

Bisher wurden drei Leitformen festgestellt. Hydrisch geformte Räume mit kleinen Zugängen gehören dem ersten Typ an. Der größte Querschnitt liegt oben bei den Zuflüssen, wo die Mischungskorrosion am stärksten ist. Er nimmt in Richtung des wegfließenden Wasser allmählich ab. Der Sackgang zeigt ein oberes Ende in Form einer im anstehenden Fels liegenden Apsis mit kleinen wasserzuführenden Öffnungen. Der Korrosionskalk (Abb. 1, 2, 3,) ist der dritte Typ.

Die Abkühlungskorrosion (Tab. 3, 4) erreicht bei hohen Konzentrationen und großen Temperaturdifferenzen beträchtliche Beträge. Allerdings stellen sich die dazu notwendigen Temperaturdifferenzen nur selten ein, so daß der Lösungseffekt im allgemeinen klein bleibt. Noch geringer ist die Wirkung der thermischen Mischungskorrosion.

\section{BIBLIOGRAPHIE}

BöGLI, A. : Probleme der Karrenbildung.--Geographica Helvetica, H. 3, 1951. - Karsthydrographische Untersuchungen im Muotatal. - Regio Basiliensis, Basel, 1960.

- Der Höhlenlehm. - Symposium international de Spéléologie, Como 1960.

- Kalklösung und Karrenbildung. - Zeitschrift für Geomorphologie, Supplementband 2: Karstmorphologie, 1960. 
Bögli, A. : Das Hölloch im Muotatal. - Leben und Umwelt, H. 19, 1963.

- Beitrag zur Entstehung von Karsthöhlen. - Die Höhle, H. 3, Wien, 1963.

- Mischungskorrosion, ein Beitrag zum Verkarstungsproblem. - Erdkunde, Archiv für wissenschaftliche Geographie, Bd. XVIII, H. 1/2, 1964.

Corber, J.: Les Karst du NE de l'Europe. - Inst. d. études rhodaniennes, Mémoires 12, Lyon, 1957.

- Erosion en terrain calcaire. - Annales de Géographie, H. 366.

Halliday, W. R.: Changing concepts of Speleogenesis. - Bulletin of the NSS, Vol. 22, USA, 1960.

Lehmann, H.: Chemismus kubanischer Karstwasser. - Briefl. Mitt. 1959.

Lehmann, O.: Hydrographie des Karstes. - Enzykl. der Erdkunde, Wien, 1932.

Moore, G. W.: Introduction to the Origin of Limestone Caves. - Bulletin NSS, USA, Vol. 22, 1960.

PıA, J.: Theorien über die Löslichkeit des kohlensauren Kalkes. - Mitt. Geol. Ges., Wien, 1953.

Sammassmann, H.: Theoretische Grundlagen der Bestimmung von aggressiver Kohlensäure in natürlichen Gewässern. - Schweiz. Archiv f. angewandte Wissenschaft und Technik, Jg. 13-14.

Tillmans, J.: Die chemische Untersuchung von Wasser und Abwasser. W. Knapp, Halle, 1932.

Trombe, F.: Traité de Spéléologie. - Paris, 1952.

Zehender, Stumm et Fischer: Freie Kohlensäure und pH von Wasser. Schweiz. Verein von Gas- und Wasserfachmännern, H. 11, 1956.

EXPLICATIONS DES PLANCHES 15 (1)-17 (3)

\section{PLANCHE 15 (1)}

Graphique 1. Courbe d'équilibre entre le carbonate de calcium et le $\mathrm{CO}_{2}$ (voir texte). Steigungsmaß TA = Inclinaison de la ligne TA.

Graphique 2. Courbe représentant, pour différentes concentrations, les variations du $\mathrm{CO}_{2}$ eq. en fonction de la température.

\section{PLANCHE 16 (2)}

Photo 1. Marmite de corrosion dans la paroi nord de la Galerie des Titans, dans le Hölloch (Suisse centrale).

Photo 2. Marmite de corrosion dans la paroi nord de la Galerie des Titans, au Hölloch. Dans la galerie inondée, l'eau venue de la fissure avec une teneur élevée en bicarbonate de calcium a corrodé la marmite; à l'état sec de la galerie, elle a formé des concrétions.

\section{PLANCHE 17 (3)}

Série de marmites de corrosion sur une diaclase avec diverses arrivées d'eau à la voûte de la Galerie des Titans, à l'embranchement de la Galerie du Polype, non loin du Bivouac I. 

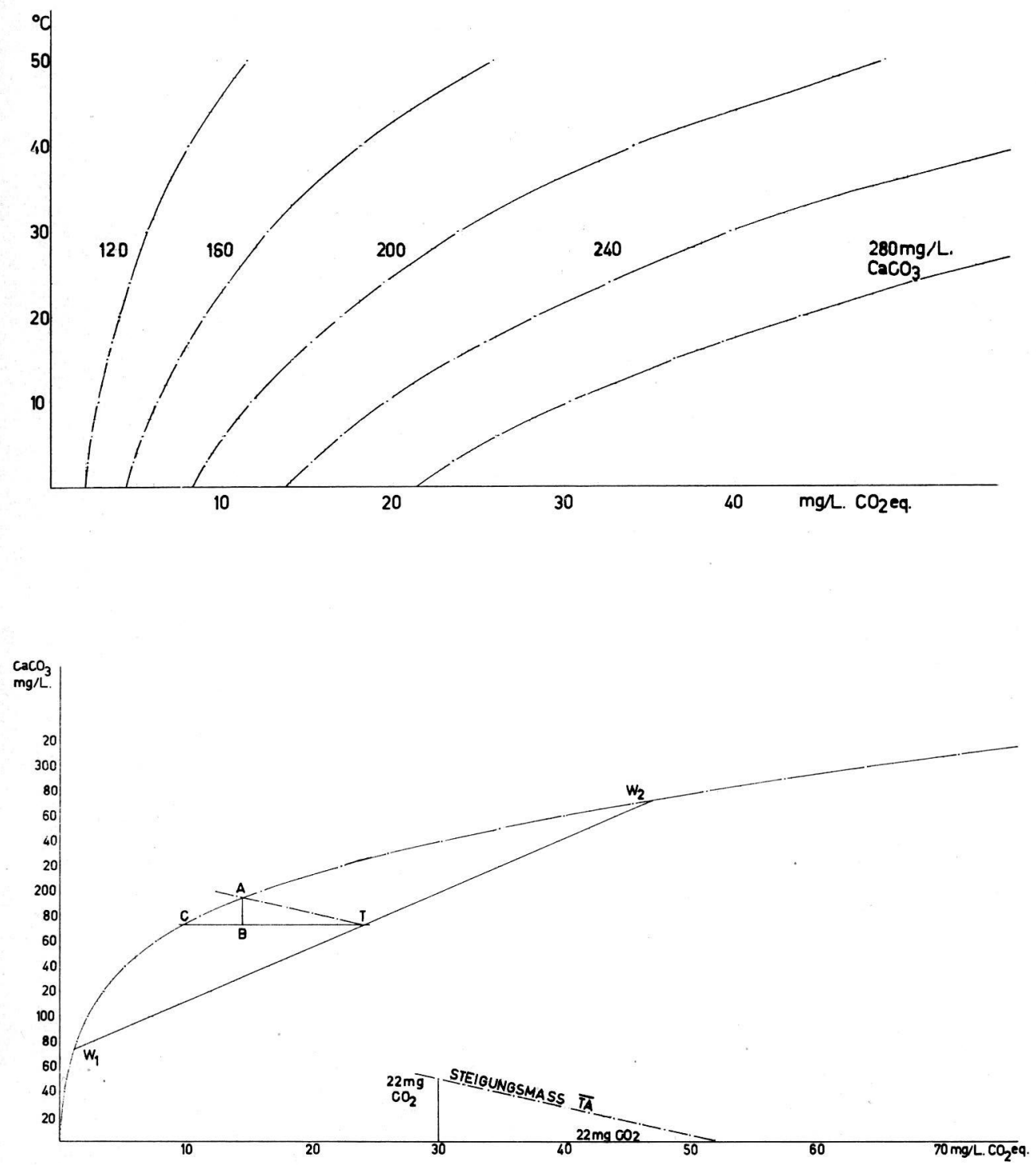

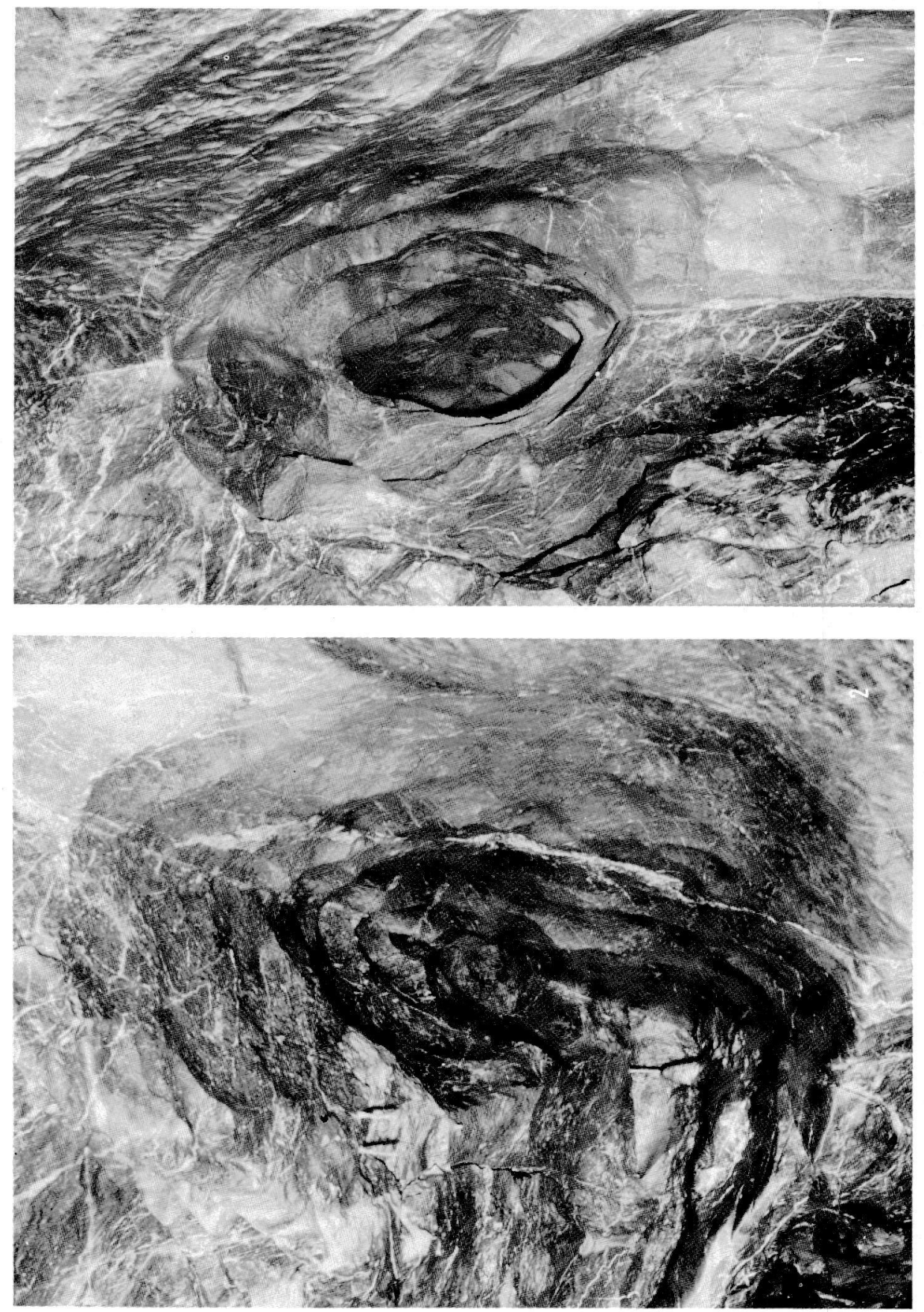


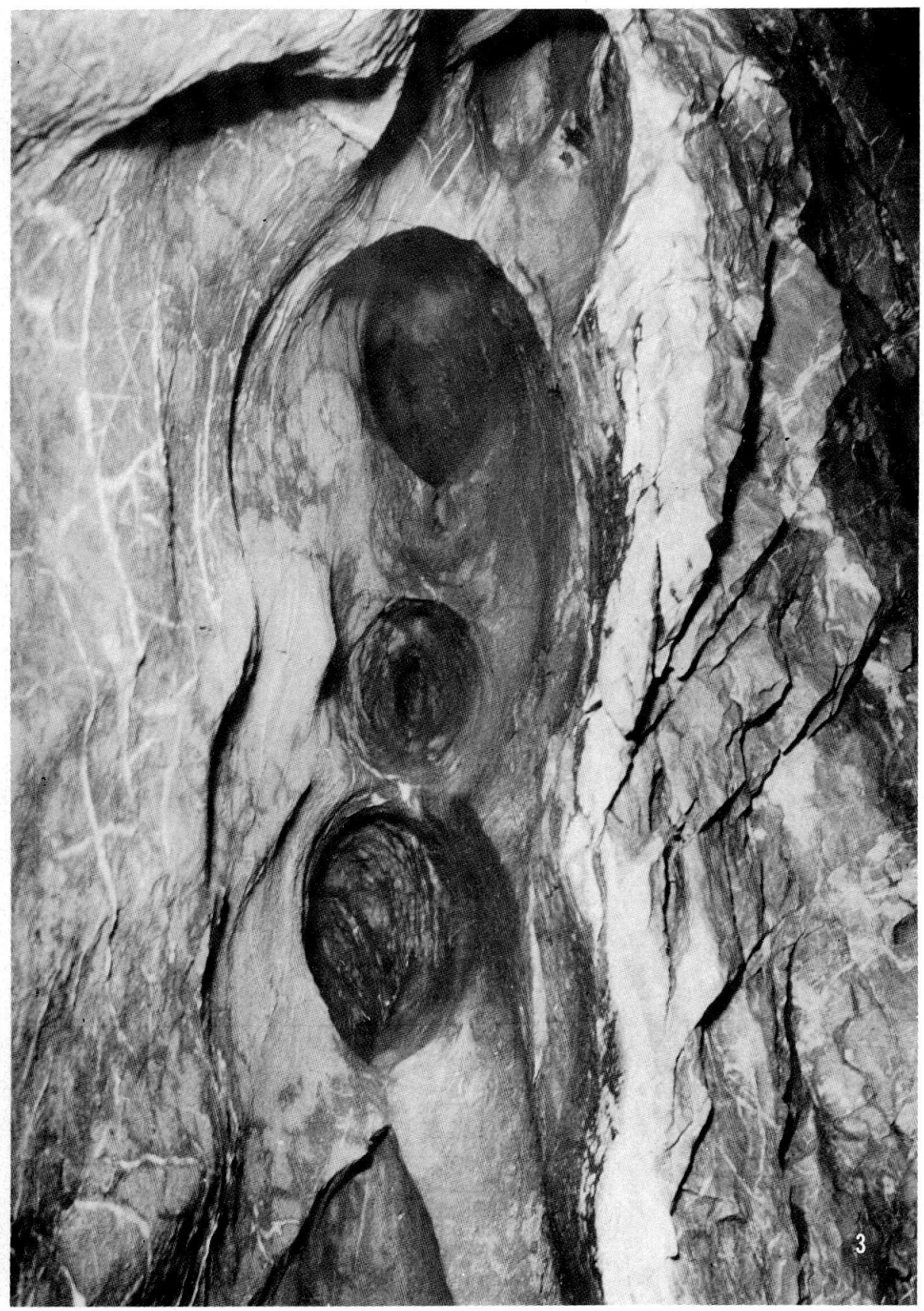

